\title{
Integrating Cortex Transform and Brightness Based Features for Multi-texture classification
}

\author{
（複数テクスチャ分類のための皮質変換と輝度特徽の総合）
}

\author{
Md. Khayrul Bashar ${ }^{\dagger}$, Noboru Ohnishi (member) ${ }^{\dagger}$
}

\begin{abstract}
This paper proposes a new scheme of fusing cortex transform and brightness based features obtained by local windowing operation. Energy features are obtained by applying popular cortex transform technique within a sliding window rather than the conventional way, while we define three features namely directional surface density(DSD), normalised sharpness index(NSI), and normalized frequency index(NFI) as mesures for pixel brightness variation. Fusion by simply vector tagging as well as by correlation is performed in the feature space and then classification is done using minimum distance classifier on the fused vectors. The interesting point is that brightness features, though inferior on some natural images, often produces smoother texture boundary in mosaic images, whereas energy features show the opposite behavior.This symmetrically inverse properties are combined through vector fusion for robust classification of muti-texture images obtained from Brodatz album and VisTex database. Results and comparison in terms of edge smoothness and confusion matrix based accuracy metrics show the robustness of the scheme.
\end{abstract}

Key words: Cortex transform, texture, mean energy, directional surface density, integration, Confusion matrix.

\section{Introduction}

Texture classification is very important in image analysis. Content based image retrieval, inspection of surfaces, object recognition by texture, OCR are examples where texture classification plays a major role. Multichannel filtering methods ${ }^{45) 6) 9) 10) 11)}$ offer potentiality and computational advantages over other methods for texture classification and segmentation. However, all the methods are highly dependent on the types of images or the imaging sensors. The particular class of techniques suitable to images from one sensor type fails for the other sensors. Also all aspects of textures are not captured by any single class of descriptors or mechanisms. Combining texture features has been suggested by some authors ${ }^{12)}$. Recently Huang et al. ${ }^{3)}$ used consensus theory to integrate deterministic and indeterministic fields of texture.

In this study, we propose to integrating features derived from multi-resolutional Cortex Transform ${ }^{7)}$, originally proposed by Goresnic et al.5) and pixel brightness variation. Though fractal features are quite effective for texture analysis, it is tedius to compute them

Received April 1, 2002; Revised July 31, 2002; Accepted September 4, 2002

† Dept. of Information Engineering, Nagoya University

(Furo-cho, Chikusa-ku, Nagoya 464-8603, Japan)

映像情報メディア学会誌 Vol. 56, No. 11,pp. 1769１778（2002 precisely. Hence we define three features namely normalized sharpness index(NSI), normalized frequency in$\operatorname{dex}(\mathrm{NFI})$ and directional surface density(DSD)as alternative to fractal dimension.

The following are the characteristics of our approach.

(1) Three simple measures, namely DSD, NSI and NFI, are formulated to characterize pixel brightness variations.

(2) In Cortex transform approach, the processing is block-based like short space Fourier transform, where the block size is fixed experimentaly. Details will be found in the reference ${ }^{15)}$

(3) Block operation, where block-size and octave scale control the total number of filters i.e., features space, eliminates two steps namely the filter selection and post-filtering transformation as $\mathrm{in}^{4)}$.

(4) Our fusion approach combines the goodness of brightness and transform features to produce more robust classification.

Section 2 briefly mentions the Cortex Transform with feature computation technique and section 3 explains the proposed brightness variability features. Section 4 describes feature integration and classification, and section 5 reveals an experimental study with the analysis of feature histograms and classifier performance. Lastly 
conclusion and future expansion of this work are discussed.

\section{Cortex Transform}

The cortex transform ${ }^{5)}$ decomposes an input image into a set of subimages according to the behaviour of simple cells in the human visual system(HVS). The band pass nature of the simple cells is modelled by the Cortex filter, which is a product of two Gaussian functions in the frequency domain, namely Radial band and Orientation filters with a radial and orientation bandwidth of 1 octave and 45 degrees respectively. In polar cordinate system, they are represented as follows.

Radial band filter:

$$
f(r)=\frac{1}{\sqrt{2 \pi} \sigma_{r}} \exp \left\{-\frac{\left(r-m_{r}\right)^{2}}{2 \sigma_{r}^{2}}\right\}
$$

Orientation filter:

$$
g(\theta)=\frac{1}{\sqrt{2 \pi} \sigma_{\theta}} \exp \left\{\frac{-\left(\theta-m_{\theta}\right)^{2}}{2 \sigma_{\theta}^{2}}\right\}
$$

Cortex filter:

$$
C(r, \theta)=f(r) g(\theta)
$$

where

$$
\begin{aligned}
m_{r} & =\operatorname{Int}\left[\frac{r_{1}+r_{2}}{2}\right], \\
\sigma_{r} & =\sqrt{\left[-\frac{\left(r_{1}-m_{r}\right)^{2}}{2 \ln \left(R_{r}\right)}\right]} \\
R_{r} & =\frac{f\left(r_{1}\right)}{f\left(m_{r}\right)} \\
r & =\sqrt{\left(u^{2}+v^{2}\right)} \\
m_{\theta} & =(\operatorname{index}) \frac{\pi}{4}+\frac{\pi}{8}, \\
\sigma_{\theta} & \left.=\sqrt{[} \frac{-\left(\frac{\pi}{8}\right)^{2}}{2 \ln \left(R_{\theta}\right)}\right], \\
R_{\theta} & =\frac{g\left(\frac{\pi}{8}+m_{\theta}\right)}{g\left(m_{\theta}\right)}, \\
\theta & =\arctan \left(\frac{v}{u}\right)
\end{aligned}
$$

Here Int [.] is a function to compute the integer value of the argument, $r$ and $\theta$ are the frequency variables and $r_{1}, r_{2}$ are the filter edge frequencies respectively. $m_{r}$, $m_{\theta}$ are means, $\sigma_{r}, \sigma_{\theta}$ are standard deviations, and $R_{r}$, $R_{\theta}$ are ratio parameters of the Gaussians respectively. 'Index' are integer values $(0,1,2,3)$ used to obtain center frequencies of an orientation filter. The frequency spectrum of a sample radial band, orientation, and cortex filter are shown in Fig.1

The detail implementation of the above Eq.(1) to (11)

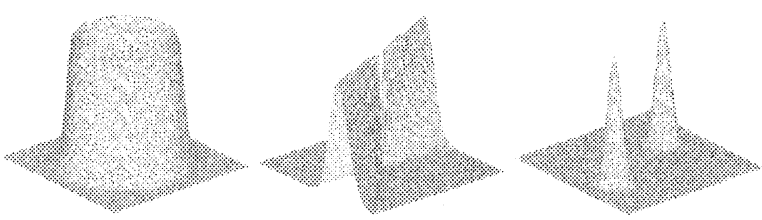

Fig. 1 The frequency spectrum of a sample (a) Radial band filter, (b) Orientation filter, and (c) Cortex filter

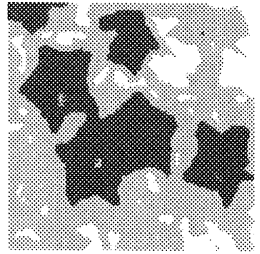

(a)

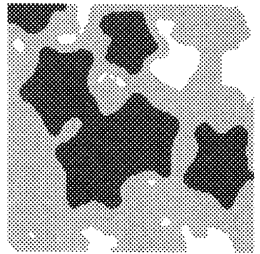

(b)

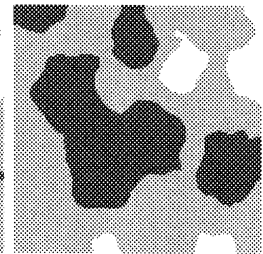

(c)
Fig. 2 Classified flower31 image (size $256 \times 256$ ) for window sizes (a) $8 \times 8$, (b) $16 \times 16$, (c) $32 \times 32$. Class boundary distorts with increasing window sizes.

are given $\mathrm{in}^{5) 15)}$. Only the feature image generation scheme is explained here for illustrating its purpose.

\section{1 Feature Image Generation}

The generation of a set of features, denoted as $\Omega_{c}=\left\{A E^{\prime} s, M D^{\prime} s\right\}$, is done by sliding block operation, where the block or window size is determined based on a boundary verification experiment over a number of images. The motivation is to compromise between resolution and stability. Based on this experiment, we decided to use a window of $16 \times 16$, which is the observed convenient size for a class of images. Fig. 2 shows how class boundary distorts gradually with the increase in window size. The number of cortex filters within a block is determined based on octave scale. This is justified by the radial frequency bandwidth of the simple cells in the visual cortex of HVS. According to this scale, the number of Cortex filters is as follows:

$$
N C F=4 \log _{2}\left\{\frac{(N / 2)}{f_{\text {int }}}\right\},
$$

where $N$ and $f_{\text {int }}$ represent an square block size, and the lowest radial frequency. For most cases, we used $f_{\text {int }}$ of 2 or 4 units. Two residual filters, covering the lowest and highest frequency regions of the block, are used to extract finest and coarsest texture information.

We compute texture features based on the avgerage energy and magnitude deviation over the filtered images as follows :

$$
E_{i j}\left(x_{0}, y_{0}\right)=\frac{1}{N^{2}} \sum_{x=0}^{N-1} \sum_{y=0}^{N-1}\left|F_{i j}(x, y)\right|^{2},
$$




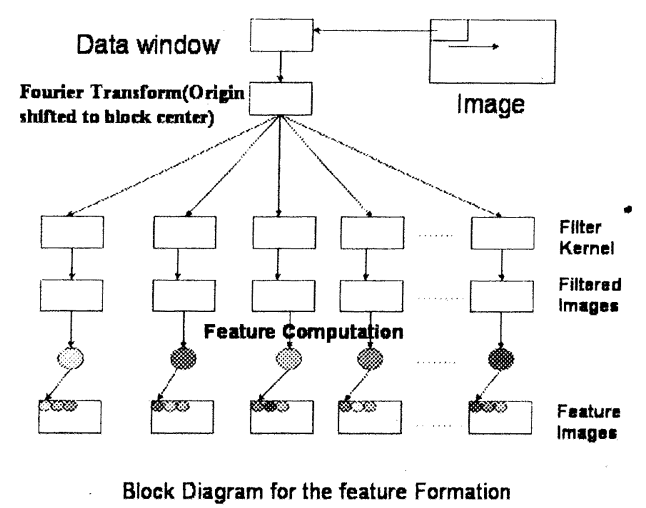

Fig. 3 A simple block diagram of statistical feature(Cortex) computation

$$
M D_{i j}^{2}\left(x_{0}, y_{0}\right)=\frac{1}{N^{2}} \sum_{x=0}^{N-1} \sum_{y=0}^{N-1}\left(\left|F_{i j}(x, y)\right|-\mu_{\left|F_{i j}\right|}\right)^{2},
$$

where $F_{i j}(x, y)$ is the filtered image-block, centered at $\left(x_{0}, y_{0}\right)$, and $\mu_{\left|F_{i j}\right|}$ is its mean in $i$ th radial band and $j$ th orientation. A simple feature computation procedure is given below(See Fig.3).

(1) Choose a square block $(16 \times 16)$ of data, compute dft magnitude and translate the transform origin to the center of the block.

(2) Compute the number of filters by Eq.12 and construct their kernels using Eq. 1 to Eq.11.

(3) Multiply the transformed data with each of the kernels, compute features using Eq.13, 14 and store them into seperate arrays.

(4) Shift the window horizontally and vertically by appropriate pixels(one pixel in our implementation) and follow steps 1 to 3 untill the whole image is scanned. This generates a set of feature images.

\section{Features Based on Pixel Brightness Variation}

\section{1 Feature Definition}

It has been reported that fractal features are useful for texture analysis ${ }^{1213) 14)}$. However, precise values of these features are difficult to compute especially for a pattern of small area. Hence we attempted to define the following features based on the pixel brightness variation over a sliding block of size $N^{2}$ centered at $\left(x_{0}, y_{0}\right)$.

Directional Surface Density(DSD) is defined as:

$$
D S D_{\Delta x, \Delta y}\left(x_{0}, y_{0}\right)=\frac{1}{N^{2}} \sum_{x=0}^{N-1} \sum_{y=0}^{N-1} \mid f(x, y)
$$

$$
-f(x+\Delta x, y+\Delta y) \mid
$$

where

$$
\begin{aligned}
& \Delta x=1,2,3, \ldots \ldots \ldots, N . \\
& \Delta y=1,2,3, \ldots \ldots \ldots, N .
\end{aligned}
$$

This feature corresponds to the directional surface value of the gray scale image when it is considered as the three dimensional object constructed by $\mathrm{x}, \mathrm{y}$, and brighness axes. It can be computed for many directions and spacing of interest by vaying the values of $\Delta x$ and $\Delta y$ and found to be effective in texture feature extraction with noise suppression and boundary preservation. Normalized Sharpness Index(NSI)is defined as:

$$
N S I\left(x_{0}, y_{0}\right)=\frac{1}{N^{2}} \sum_{i} S B C_{i}
$$

where $S B C_{i}= \begin{cases}1 & \text { if } i \in \Omega, \\ 0 & \text { elsewhere }\end{cases}$

Here $\Omega=\left\{S_{k}\right\}$, is a set of all discrete points, where a condition $f\left(S_{k-1}\right)-f\left(S_{k}\right)>t h$ is satisfied. $S_{k}$, a smallest positive integer beyond $S_{k-1}$, can be defined as $S_{k}=\sum_{i=1}^{k} n_{i}$ where $S_{0}=\sum_{i=1}^{0} n_{i}=0, n_{i}$ 's are the spatial co-ordinate values at the satisfactory points, and $f($.$) is a 1 \mathrm{D}$ version of the image. This feature indicates the sharpness in the brightness changes. However, proper threshold selection is important. We used $15 \%$ of the dynamic range of the image as threshold value. Actually 1D representation of image blocks may represent varities of shapes even for the same texture due to various lighting conditions, sensor power, noise, distance and direction between sensor and scene etc. What we are trying by this threshold is to extract sharpness and frequency of brightness changes in a small region. For a specific 1D signal, higher values of NSI are expected in the sharp-changing part, while lower values for the smoother part. A suitable threshold will serve the purpose. However the above choice is only a reasonable trial value. Practical knowledge on the sensor signals (under various conditions) for different texture types would be more helpful. It may lead to extend an automatic method of fixing proper threshold value.

Normalized frequency index(NFI)is defined as:

$$
N F I\left(x_{0}, y_{0}\right)=\frac{1}{N^{2}} \sum_{i} F B C_{i}
$$

where $F B C_{i}= \begin{cases}1 & \text { if } i=S_{k-1} \in \Omega, \\ & \text { and } D I F F_{k} * D I F F_{k+1}<0, \\ 0 & \text { elsewhere }\end{cases}$ 
Here $D I F F_{k}=f\left(S_{k-1}\right)-f\left(S_{k}\right)$ or $f\left(\sum_{i=1}^{k-1} n_{i}\right)-$ $f\left(\sum_{i=1}^{k} n_{i}\right)$ and $k=1,2, \ldots, k_{\max }$

This feature indicates the frequency of the brightness changes. This is also an effective feature for texture characterization for an appropriate threshold value.

\section{2 Feature Computation}

The above brightness features, denoted as $\Omega_{b}=$ $\left\{N S I, N F I, D S D^{\prime} s\right\}$, are computed directly from a rectangular sliding window centered around each pixel of an image. It is important to use larger windows(area less than the smallest texture class) to grow the texture features more reliably and to remove unnecessary noises. However, larger window oversmooths various texture classes and produces double boundary among the inter-texture classes. To minimize the effect, we used an square window size of 13 to 17 for the images of interest.

\section{Integration and Classification}

\section{1 Integration}

In our approach, we used feature level integration through combining various candidate features into two ways namely vector fusion and correlation co-efficient based fusion guided by fuzzy logic literature as $i^{16)}$

In the vector fusion method, we just compute feature vectors from different approaches, for example cortex transform and brightness measures. We then put them sequentially to obtain a fused vector at each spatial point. Fused vector corresponding to each pixel is compared with the class representative fused vectors to obtain classified images.

In the second approach, we are motivated by fuzzy logic, where we devided the derived features into "similar", "dissimilar", and "as it is" groups based on the threshold $(0.90,0.25)$ of the feature correlation coefficients are computed using Eq. 18.

$$
r=\frac{\operatorname{Cov}\left(f_{m} f_{n}\right)}{\sqrt{\operatorname{Var}\left(f_{m}\right) \operatorname{Var}\left(f_{n}\right)}}
$$

where $f_{m}, f_{n}$ are the feature images of the two feature groups such that $f_{m} \in \Omega_{c}$ and $f_{n} \in \Omega_{b}$.

Then we used 'AND' and 'OR' logic among the similar and dissimilar features to obtain the fused features. The fusion rules are as follows:

Rule 1: if $r \geqq 0.90$, features are similar. So the fused feature $=$ MIN $($ feature1, feature2, featureN).

Rule 2: if $r \leqq 0.25$, features are dissimilar. So the fused feature $=\operatorname{MAX}($ feature1, feature2,.....,featureN).

However we left the rest of the features in "as it is"

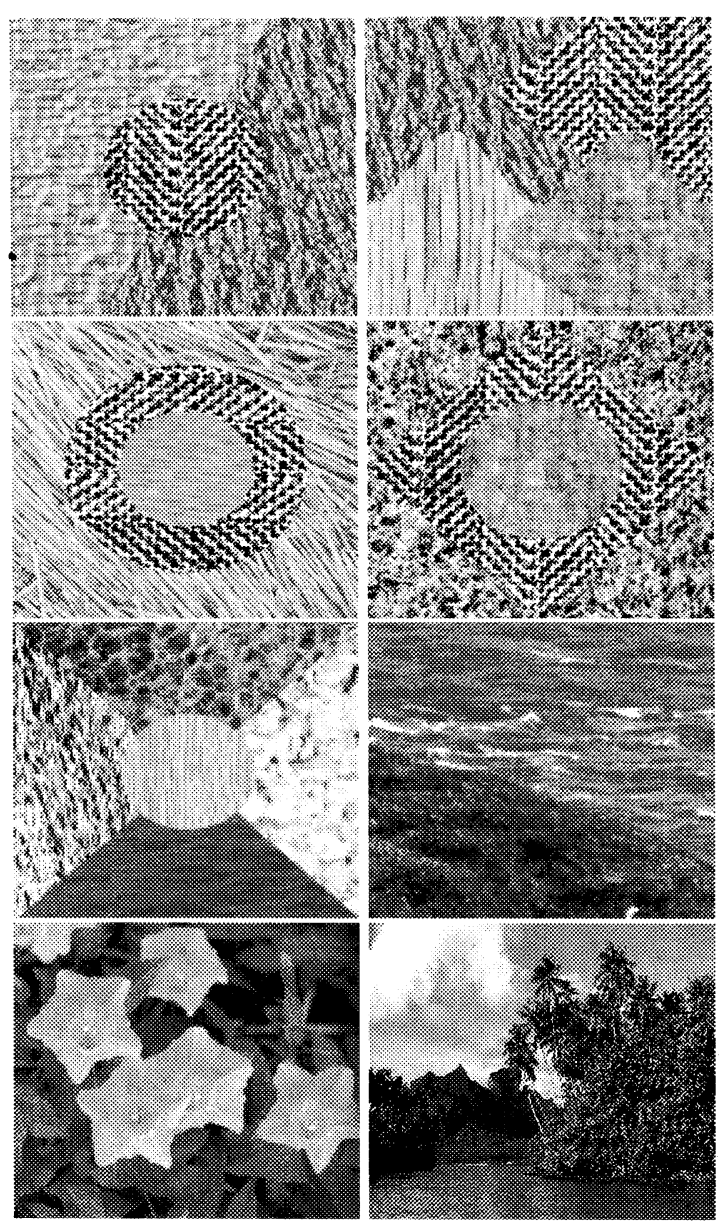

Fig. 4 Some sample images(mosaic, natural scene, size $256 \times 256$ ) from Brodatz album and VisTex database.

group untouched and directly applied to the classifier.

\section{2 Classification}

Classification of the fused features is performed in supervised manner using minimum distance classifier that uses Euclidean distance as similarity measure. In the training phase, we select some samples from each texture class of the input image by visual inspection. Then all the features(cortex and brightness) are computed as explained in section 2 and 3 . In order to obtain representative class vectors, we just average out the feature vectors of the samples from each class and stack them together. The training vectors so formed and the fused feature vector, corresponding to each image co-ordinate, are inputted to the classifier. Classifier iteratively labels each pixel, based on the minimum distance criterion. Two sample feature vectors for Cortex transform and brightness based features are as follows:

$$
\begin{aligned}
& V_{c}(x, y)=\left[E_{i j}(x, y), M D_{i j}(x, y)\right] \\
& V_{b}(x, y)=\left[N S I(x, y), N F I(x, y), D S D_{\Delta x \Delta y}(x, y)\right]
\end{aligned}
$$

where 


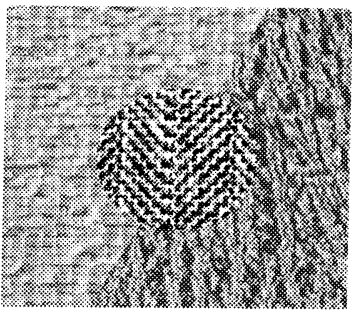

(a)

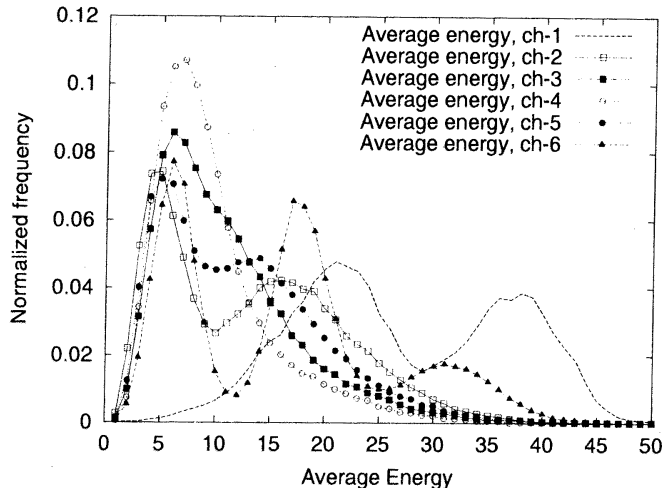

(c)

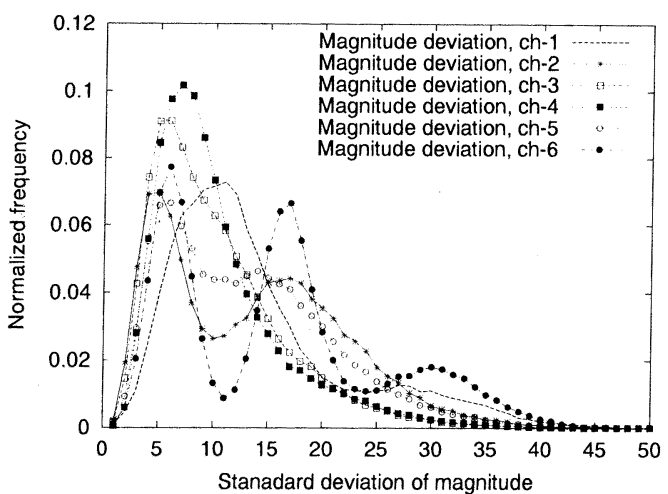

(d)

Fig. 5 Results of Cortex Transform based features. (a) Original mos31 image, (b) Classified image, Histogram of(c)the average filtered energy, (d)the magnitude deviation of filtered image.

$$
\begin{aligned}
i & =1,2,3, \ldots ., S . \\
j & =1,2, \ldots, d_{\max } . \\
\Delta x & =1,2, \ldots \ldots, R . \\
\Delta y & =1,2, \ldots \ldots ., C .
\end{aligned}
$$

Here $\mathrm{S}$ is total scales and $d_{\max }$ indicates total orientations.

\section{Experimental Results and Discussions}

We conducted an experiment on the two groups of images obtained from the standard Brodataz texture album and also from VisTex database in the internet. Twenty mosaic images from Brodatz album and thirty natural images from VisTex, all having (256x256) pixple texture images are shown in Fig.4. Table 2 shows els, were examined in the experiment. Some of the sam-

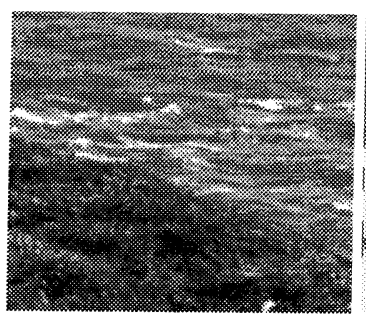

(a)

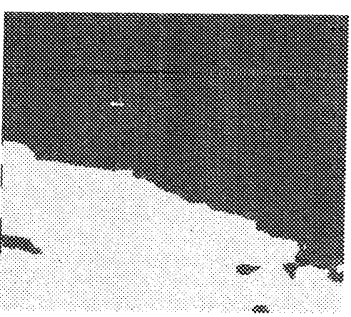

(b)

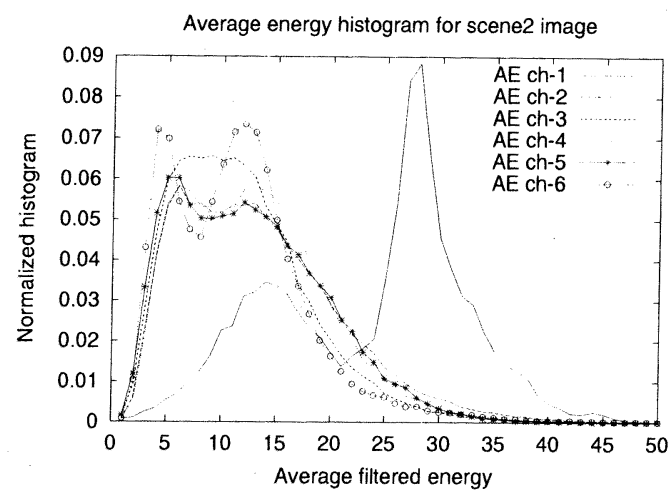

(c)

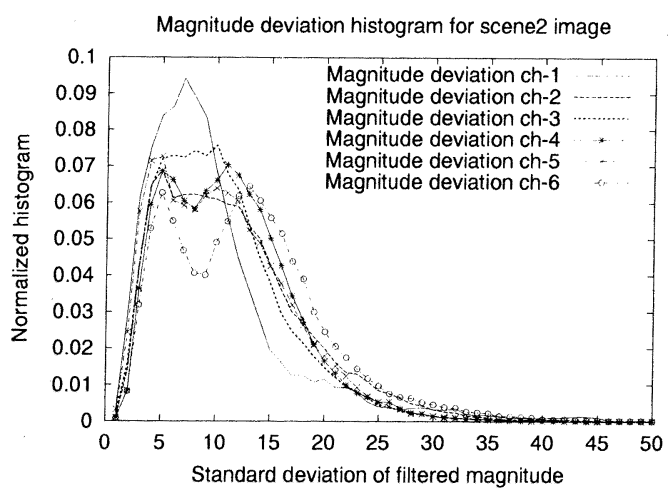

(d)

Fig. 6 Results of Cortex Transform based features. (a) Original scene21 image, (b) Classified image, Histogram of (c) the average filtered energy, (d) the magnitude deviation of filtered image. the names of 20 images from both groups, where first subscript indicates number of expected classes and second subscript represents number of images for a particular first subscript.

\subsection{Results}

(1) Using Cortex Transform Based Features

We performed classification using Cortex filtering approach as explained in section 2 . We used a maximum of two scales i.e., two center frequencies of 3 and 6 units with intial radial frequency, $f_{\text {int }}$ of 2 units. The suitable value of the filter passband controlling parameter ratio, $R_{r}$ or $R_{\theta}$ was found to be 0.01 to 0.03 . Figs.5 and 6 indicate two results of classification with corresponding slotted feature histograms. The multiple peaks of the histograms clearly indicates the potentiality of the cortex filtering approach. It is worthy to mention that 


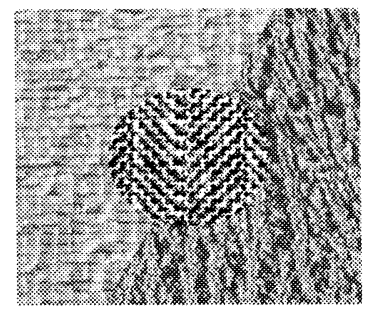

(a)

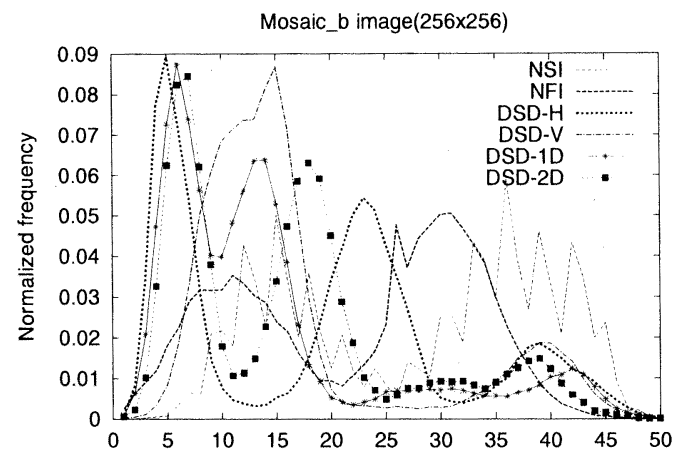

Normalized sharpness and frequency index(NSI,NFI), Directional surface density(DSD)

(c)

Fig. 7 Results of pixel brightness based features.

(a) Original mos31 image, (b) Classified image,

(c) Histogram of the DSD features in four directions.

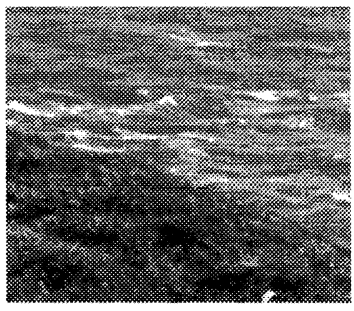

(a)

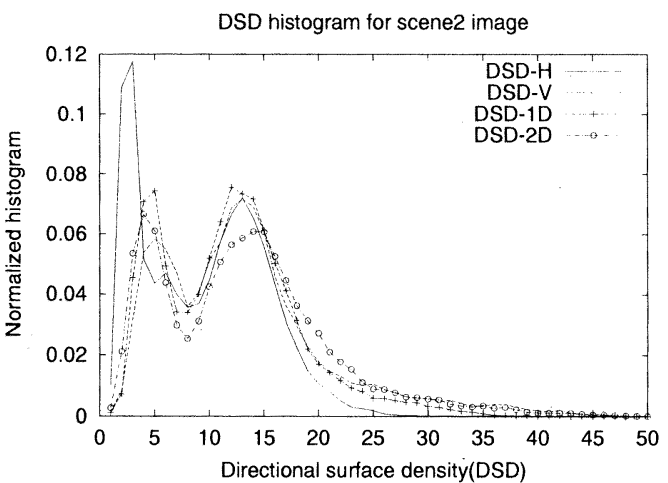

(c)

Fig. 8 Results of pixel brightness based features.

(a) Original scene21 image, (b) Classified image,

(c)Histogram of the DSD features in four directions.

feature histograms were calculated after deviding the entire feature range into fixed number of slots namely 50. In all the images, we used an window size of $16 \times 16$ as explained in section 2. However, class boundaries are not smooth enough in case of Brodatz images.

(2) Using Pixel Brightness Based Features

The intensity images are used directly to compute DSD, NSI, NFI features those extract pixel brightness variations from all of the 50 images. However, experiences show that the minimum window size should be $13 \times 13$ for the kind of images we used. This size appears necessary and imprtant to grow the above brightness features for reliable texture representation. These features extract smoother boundary with noise suppresssion in case of Brodatz mosaic textures as shown in Fig.7 with corresponding slotted histograms. These histograms demonstrate that the number of peaks are approximately equal to the actual texture classes in images. However, for some natural scene images, for example scene21 image as in Fig.8, they fail to aggregate the homogenous texture elements.

\section{(3) Using Combined Features}

Integrating features eliminates the problems in the above methods. From the Fig. 6(c) and (d), it shows, though all features(AE,MD) from ch- 2 to ch-6 are highly overlapped around 0-25 slots, the low frequency channel(ch-1) features (AE) have uncorrelated peaks (solid lines) in the feature space compared to those in brightness features as shown in Fig. 8(c), where all the surface density features appears in phase. For the three class problem in mos31 image as shown in Figs. 5 and 7, both the descriptors(cortex and brightness) well classify the three classes and the corresponding histograms also reveal more or less similar correlation. So for better classification, minimal correlation among various features with relatively higher feature values are expected.

From this obsevation we decided to fuse these two kind of features to obtain more robust classification. We attempted two techniques, namely vector fusion and correlation co-efficient based fusion as explained in section 4. Figs. 9-14 show the performance of our method before and after vector fusion. From the scene51 image as shown in Fig.10, it is observed that brightness features fails to extract cloud(top-left corner) and water(bottom), whereas the cortex transform based and combined features have extracted these classes successfully.

Fig.15 shows a visual comparison of the classified images obtained from vector fusion and correlation fusion technique. Clearly vector fusion approach appears better.

\section{(4) Performance Study}

The performance of our integration technique is analyzed by the following criteria namely (a) Edge Smoothness Index(ESI), and (b) Confusion Matrix(CM).

Edge Smoothness Index(ESI): 


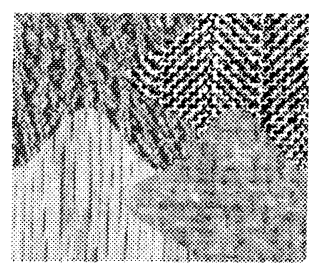

(a)

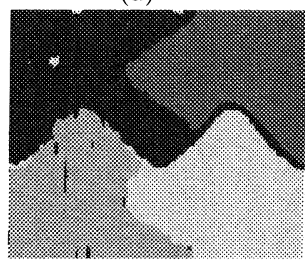

(c)

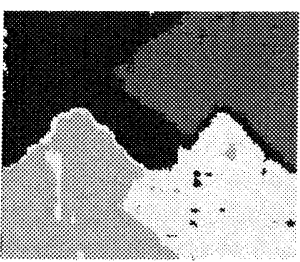

(b)

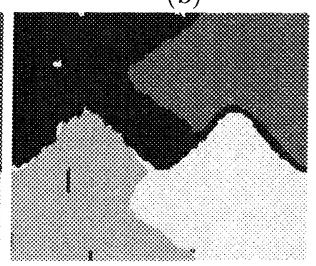

(d)
Fig. 9 Results before and after vector integration (a) Original mos41 image, Classified images of (b)Cortex,(c) brightness, and (d) combined features.

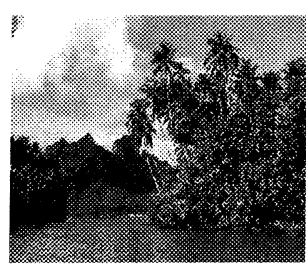

(a)

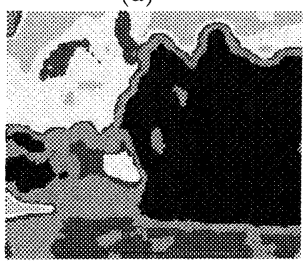

(c)

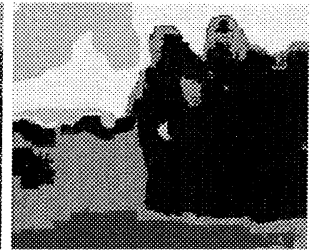

(b)

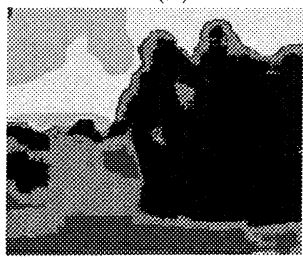

(d)
Fig. 10 Results before and after vector integration.

(a) Original scene51 image, Classified images of

(b) cortex,(c) brightness, and (d) combined features.

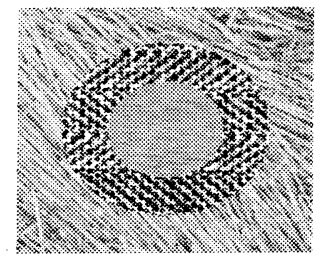

(a)

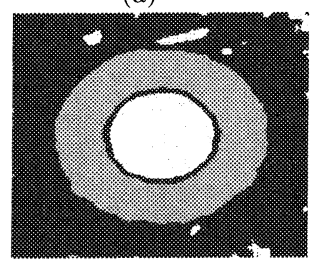

(c)

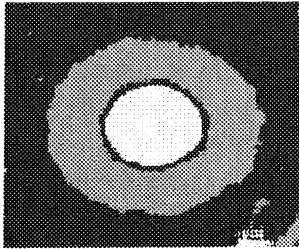

(b)

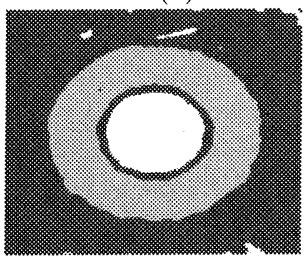

(d)
Fig. 11 Results before and after vector integration. (a) Original mos32 image, Classified images of (b) cortex,(c) brightness, and (d) combined features.

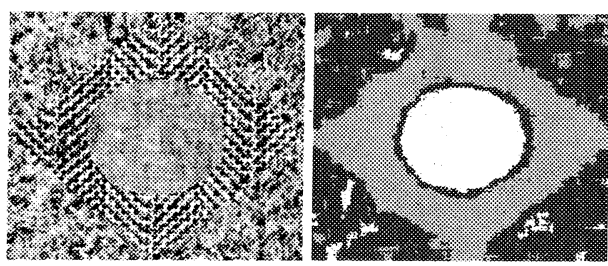

(a)

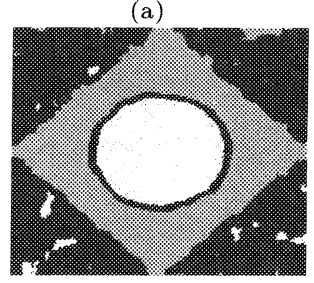

(c)

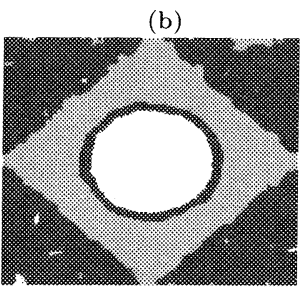

(d)

Fig. 12 Results before and after vector integration.

(a) Original mos33 image, Classified images of

(b) cortex,(c) brightness, and (d) combined features.

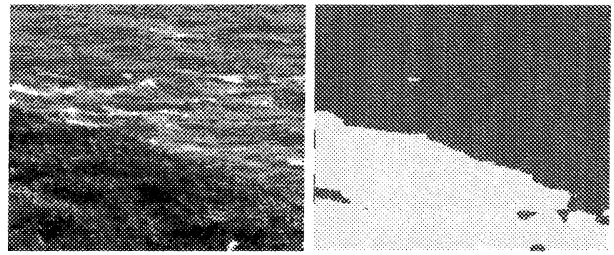

(a)

(b)
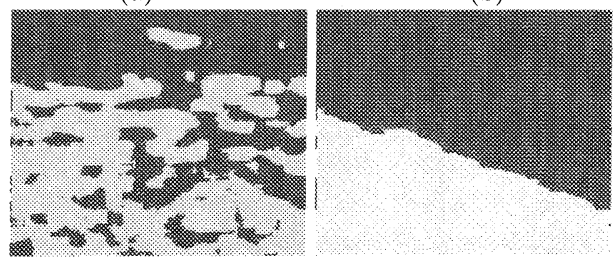

(d)

Fig. 13 Results before and after vector integration.

(a) Original scene21 image, Classified images of

(b) cortex,(c) brightness, and (d) combined features.

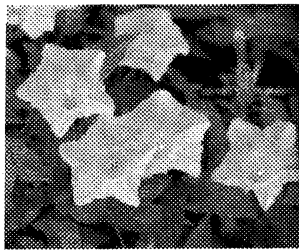

(a)

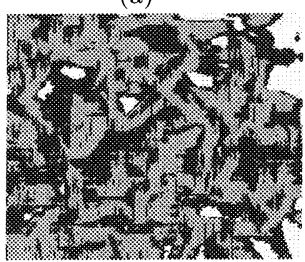

(c)

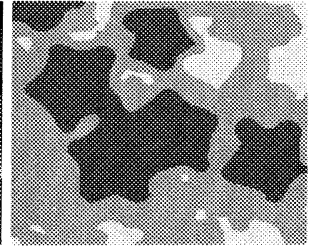

(b)

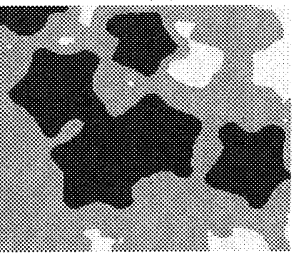

(d)

Fig. 14 Results before and after vector integration.

(a) Original flower31 image, Classified images of

(b) cortex,(c) brightness, and (d) combined features.

where, $\quad m=\sum_{i} G A_{i}$

boundary pixels obtained from the classified images.

$$
E S I=\frac{m}{N} \times 100 \%
$$




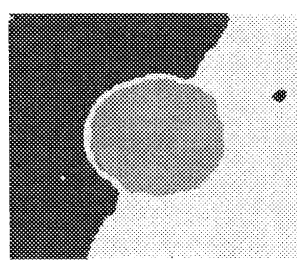

(a)

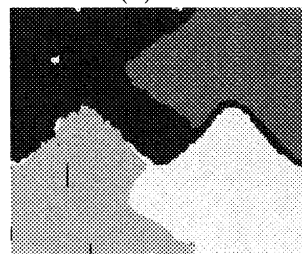

(c)

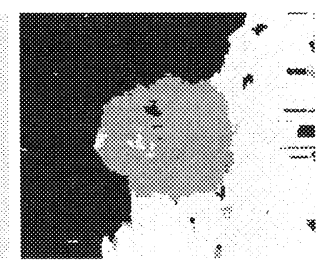

(b)

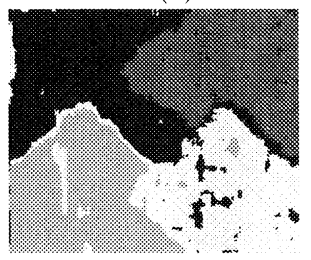

(d)
Fig. 15 Visual comparison of classified mos31 and mos41 images by our integration methods (a) and(c)stacked vector, (b)and(d)correlation method

\begin{tabular}{l|l|l|l} 
Table 1 & \multicolumn{3}{l}{ Comparison of edge smoothness measure } \\
\hline \hline \multirow{2}{*}{ Images } & Edge Smoothness Index,ESI $(\%)$, \\
\cline { 2 - 4 } & Brightness & Cortex & Combined \\
\hline mos31 & 75.76 & 71.16 & 74.55 \\
$\mathrm{~N}(\mathrm{~m})$ & $1419(\mathbf{1 0 7 5})$ & $1411(\mathbf{1 0 0 4})$ & $1230(\mathbf{9 1 7})$ \\
\hline mos32 & 79.88 & 73.09 & 78.97 \\
$\mathrm{~N}(\mathrm{~m})$ & $1993(\mathbf{1 5 9 2})$ & $2824(\mathbf{2 0 6 4})$ & $1888(\mathbf{1 4 9 1})$ \\
\hline mos33 & 71.12 & 69.52 & 70.94 \\
$\mathrm{~N}(\mathrm{~m})$ & $2296(\mathbf{1 6 3 3})$ & $5109(\mathbf{3 5 5 2})$ & $2240(\mathbf{1 5 8 9})$ \\
\hline mos41 & 85.90 & 81.54 & 84.69 \\
$\mathrm{~N}(\mathrm{~m})$ & $1525(\mathbf{1 3 1 0})$ & $2774(\mathbf{2 2 6 2})$ & $1385(\mathbf{1 1 7 3})$ \\
\hline
\end{tabular}

$$
\begin{aligned}
\Delta \alpha_{i} & =\alpha_{i}-\alpha_{i+1} \\
\alpha_{i} & =\tan ^{-1}\left(\frac{G y_{i}}{G x_{i}}\right) \\
i & =1,2,3, \ldots \ldots \ldots, N-1 .
\end{aligned}
$$

Here $N$ is the total number of consecutive boundary pixel-pairs, $m$ is the number of smooth pixel-pairs satisfying the above gradient threshold, and $\alpha_{i}, G x_{i}, G y_{i}$ are the gradient angle and magnitudes along the $\mathrm{x}$ and $\mathrm{y}$ directions respectively. We set the gradient threshold, $\alpha_{t h}$ as $45^{\circ}$. In our computation, we first compute classboundary pixels from the classifed images using Sobel edge detectors and then gradient angles and subsequent angle difference $\Delta \alpha_{i}$, are computed from the original images corresponding to those boundary pixels. Fig.16 shows the class boundaries for mos31 and mos32 images, whereas Table 1 shows a comparison of ESI's among four mosaic images (mos31, mos32, mos33 and mos41). These results indicate the smoother boundaries for the brightness features compared to cortex features. Note that the boundaries for the combined feature approximate the performance of smoother category.

Confusion Matrix(CM): We compute classification accuracies based on the concept of well known con-

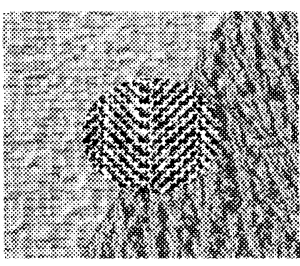

(a)

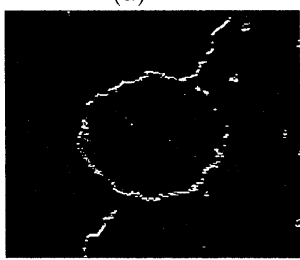

(c)

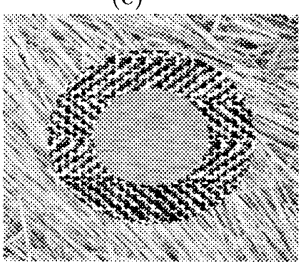

(a)

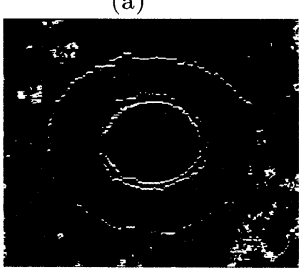

(c)

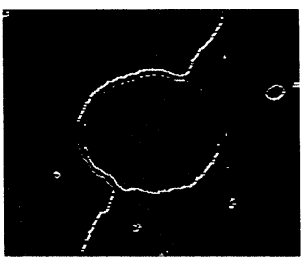

(b)

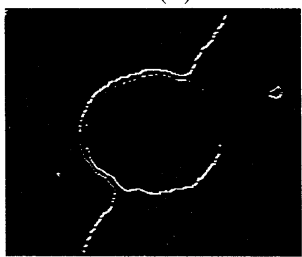

(d)

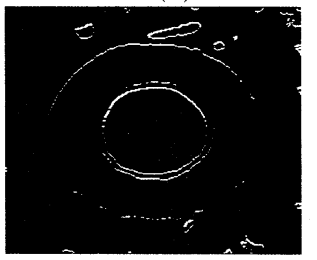

(b)

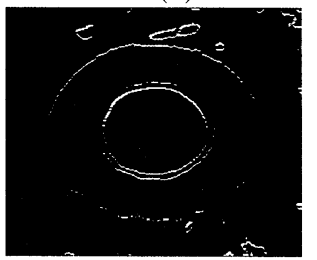

(d)
Fig. 16 Classified boundary (a) Original images $(\operatorname{mos} 31, \operatorname{mos} 32)$, edge images from(b) Cortex features, (c) brightness features, and (d) Combined features.

fusion matrix $C_{i j}$, a square matrix whose diagonal elements determines what percentage of the original class pixels are accurately classified by the classifier. The matrices are formed by matching labels of the user defined true samples with the corresponding samples estimated by the classifier. The accuracy measures are quantified by individual and overall class accuracy defined as:

$$
\begin{aligned}
I C A_{i} & =\frac{C_{i i}}{\sum_{j=1}^{n} C_{i j}} \\
O A & =\frac{\sum_{i=1}^{n} C_{i i}}{\sum_{i=1}^{n} \sum_{j=1}^{n} C_{i j}}
\end{aligned}
$$

Here $i, j=1,2, \ldots, n$. denote various classes and $n$ is the number of expected classes in an image. Conventionally, the error matrix and accuracy are computed over a large number of test samples per texture category. However, we are focusing on the classification of multi-textured images. Therefore we applied them over some disjoint samples(maximum three samaples, each of size $16 \times 16$, per class) per texture category from within an original multi-textured image. In our supervised method, user fixes the sample size and location based on his interpretation skill through careful visual 
Table 2 Comparison of overall accuracy among cortex, brightness, and fused approaches

\begin{tabular}{l|l||l|l|l}
\hline \hline \multirow{2}{*}{$\begin{array}{l}\text { Indices } \\
\text { and names }\end{array}$} & \multirow{2}{*}{$\begin{array}{l}\text { Sample } \\
\text { pixels }\end{array}$} & \multicolumn{3}{|c}{ Overall accuracy (\%) } \\
\cline { 2 - 5 } 1. $\operatorname{mos} 21$ & 2304 & 96.3 & 97.3 & 99.2 \\
2. mos22 & 2304 & 97.2 & 97.0 & 100.0 \\
3. mos23 & 2304 & 95.8 & 96.5 & 98.5 \\
4. mos31 & 2304 & 97.4 & 97.3 & 99.2 \\
5. mos32 & 2304 & 98.5 & 97.4 & 98.9 \\
6. mos33 & 2304 & 95.5 & 97.4 & 97.7 \\
7. mos41 & 3072 & 94.8 & 95.8 & 97.5 \\
8. mos42 & 3072 & 95.3 & 96.5 & 97.8 \\
9. mos51 & 2560 & 97.6 & 64.6 & 99.6 \\
10. mos52 & 2560 & 95.0 & 75.7 & 98.3 \\
11. scene51 & 2560 & 92.0 & 87.6 & 95.2 \\
12. scene21 & 2560 & 93.2 & 53.6 & 98.4 \\
13. scene31 & 2560 & 92.3 & 70.6 & 97.1 \\
14. cloud31 & 1536 & 96.3 & 68.4 & 98.0 \\
15. cluod21 & 1536 & 94.8 & 73.2 & 98.6 \\
16. Flower31 & 1536 & 98.9 & 50.2 & 100.0 \\
17. Flower21 & 1536 & 98.6 & 58.3 & 100.0 \\
18. Build.31 & 1536 & 91.8 & 77.6 & 94.1 \\
19. Build.32 & 1536 & 89.8 & 73.6 & 93.0 \\
20. Build.33 & 1536 & 88.6 & 68.3 & 93.2 \\
\hline
\end{tabular}

Table 3 Average overall accuracy

\begin{tabular}{l|l|l}
\hline \hline Methods & \multicolumn{2}{|l}{ Avg. overall accuracy (\%) } \\
\cline { 2 - 3 } & 20 images & 50 images \\
& 10 Mosaic & 20 mosaic \\
& 10 natural & 30 natural \\
\hline Cortex & 94.9 & 90.7 \\
Brightness & 79.8 & 62.5 \\
Combined & 97.7 & 93.4 \\
\hline
\end{tabular}

inspection of the original images. Table 2 shows the overall classification accuracy of 20 (10, Brodatz and 10, VisTex) images out of a total of 50 images. The average overall accuracy of the complete image group and its sub-set are summerised in table 3. Fig. 17 reflects the performance comparison before and after feature integration. This shows our combined method attains the goodness of cortex features and improves the average accuracy by a narrow margin.

\section{2 Discussions}

Statistical features like mean energy and magnitude deviation apprear robust for natural images including micro-textures as in scene21 image. Despite unsmooth boundary in mosaic images, they show better discrimination ability on both image types. Our block-based processing reduces feature space dimensions to 10 for a maximum scale usage to 3 . The use of fewer scales, fixed by the window size and octave spacing as determined by the 'boundary verification experiment', appears reasonable for the classes of images(mosaic and natural) considered in our experiment. However larger window (more than 16) rapidly increases computation time in our pixel based approach. One solution to this problem,

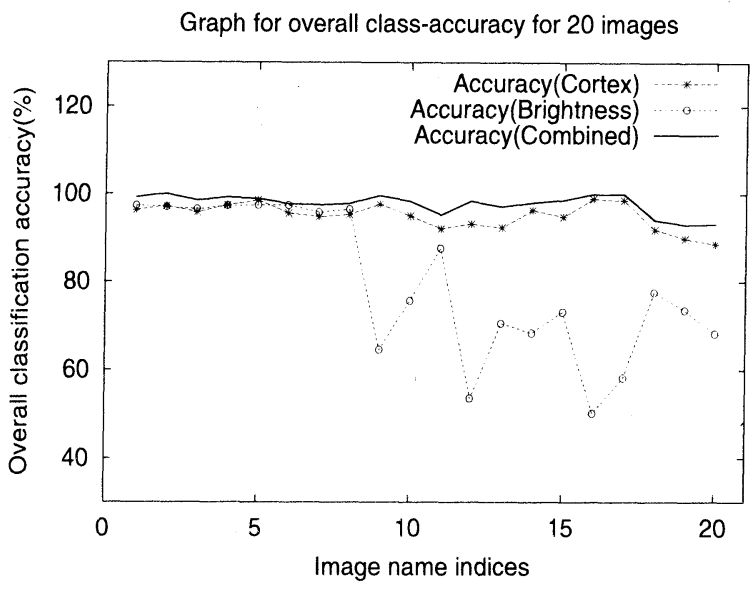

Fig. 17 Graph showing the performance of our feature integration

is to shift the window by more than one pixel.

Pixel brightness based approach is computationally feasible even for pixel operation, as it does not require Fourier transform. Generally they produce smoother boundary compared to satatistical features. However they fail to aggregate pixels in the low textured images like scene21 and flower31 images. The reason lies in the inability of brightness features to produce uncorrelated or minimal correlated features. The thereshold for these features is determined as a reasonable trial value (15\% of the dynamic range of an image). Because the magnitude of $1 \mathrm{D}$ signal obtained from a small texture region depends on many factors like texture types, sensor power and noise, distance and direction between sensor and scene etc.

During computation of ESI, we used a gradient threshold of $45^{\circ}$. In general, the lower the threshold value, the tighter the smoothness constraint. However its proper value depends on the expected degree of smoothness, which largely depends on applications.

\section{Conclusion}

A novel approach of image texture classification has been proposed. The main observation is that all aspects of texture can not be captured by a particular category of techniques or features. Hence fusing different types of features is inevitible. Though in mosaic texture images, boundary appears unsmooth, our cortex transform based statistical features better capture the textural features including microtextures. On the contrary, brightness based features like DSD, NSI, and NFI produce smoother boundary with computational adavantage but they fail to aggregate pixels in 
some natural scene images. Fusing features of different categories often integrates the goodness of individuals to produce robust classification as shown for mos31, $\operatorname{mos} 32, \operatorname{mos} 33, \operatorname{mos} 41$, scene51, scene21, flower31 images. However, vector fusion appears better than correlation based fusion in our preliminary studies. More investigation is necessary, to conclude the robustness of the correlation based fusion technique. So the future subject is to conduct more analysis on the feature correlation and integration with application to multisensor images. We also investigate the performance of our method under various experimental conditions.

\section{Acknowledgements}

We would like to thank Dr. H. Kudo, Dr. T. Matsumoto, and Dr. Y. Takeuchi for their valuable comments during the research in progress. We also thankful to Mr. T. Mizuno for his assistance during manuscript preparation. Part of this research has been supported by the Hori Science Information Foundation, Japan.

\section{[References]}

1) A. H. S. Solberg," Texture fusion and classification based on flexible discriminany analysis. In Proceedings of 13th IEEE International Conference on Pattern Recognition, 2, pp.596-600, 1996

2) A. H. S. Solberg and A. K. Jain, "Texture fusion and feature selection applied to SAR imagery", IEEE Transactions on Geoscience and Remote Sensing, 35, 2, March 1997.

3) Y. Huang, and K. L. Chan, "Multi-model Feaure Integration for Texture Analysis", In Proceedings of the 5th Asian Conference on Computer Vision (ACCV), Melbourne, Australia, 2, pp.556561,Jan.22-25, 2002.

4) Anil K. Jain and Farshid Farrokhnia, "Unsupervised Texture segmentation using Gabor Filters", Pattern Recognition, 24, 12, pp.1167-1186, May 1991.

5) C. Goresnic, and S.R. Rotman, "Texture Classification Using the Cortex Transform", CVGIP:Graphical Models and Image Processing, 54, 4, pp.329-339, July 1992 .

6) Dennis Dunn and William E. Higgins, "Optimal Gabor Filters for texture segmentation", IEEE Trans. Image Processing, 4, 7, pp.947-963, July 1995.

7) A. B. Watson, "The Cortex Transform: Rapid Computation of Simulated neural images", Comput. Vision Graphics Image Process., 39, pp.311-327, 1987.

8) M. pasaresi, "Texture Analysis for Urban Pattern Recognition using Fine-resolution Panchromatic Satellite Imagery", Geographical and Environtal Modelling, 4, 1, pp.43-63, 2000.

9) Zhu, C., and X. Yang, "Study of Remote Sensing Image Texture Analysis and Classification using wavelet", International Journal of Remote Sensing, 13, pp.3167-3187, 1998.

10) Chun S. Lu, Pau C. Chung and Chih F. Chen, "Unsupervised Texture segmentation via Wavelet Transform", Pattern Recognition, 30, 5, pp.729-742, 1997.

11) P. Scheunders, "A multi-valued image wavelet representation based on multi-scale fundamental forms", IEEE Trans. on Image Processing, to appear 2001

12) A. P. Pentland, "Fractal based description of natural scenes,IEEE Trans. Pattern Analysis Mach. Intell., PAMI-9, pp.661-674, Nov., 1984

13) Chen C.C., J.S. Daponte, and M.D. Fox, "Fractal feature Analysis and Classification in Medical Imaging", IEEE Transaction on Medical Imaging, 8, pp.133-142, 1989.

14) Super, B.J. and A.C. Bovik, "Localized measurement of Image Fractal Using Gabor Filters," Journal of Visual Communication and Image Representation, 2, pp.114-128, 1991.

15) M.K. Bashar, B.K.Mohan, R.K.Shevgaonkar, N.Ohnishi, "Unsupervised Texture classification of Images using Cortex Filters",
The proceedings of the fifth Asian Conference on Computer Vision (ACCV), Melbourne, Australia, 2, pp.828-833, Jan.22-25, 2002.

16) P. J. Deer, and P. W. Eklund, "On the Fusion of Image Features", NEC Research Index found in http://citeseer.nj.nec.com/162546.html

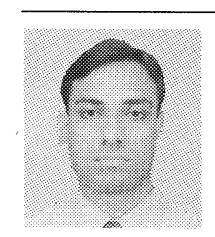

Md. Khayrul Bashar He received bachelor degree(1993) in Electrical and Electronic Engineering from Bangladesh University of Engineering and Technology(BUET) and Master of Technology(M.Tech., 1998) in Communication Engineering from Indian Institute of Technology(IIT) Bombay, India. He is currently a Ph.D. student in the department of Inforformation Engineering, Nágoya University, Japan. He is a memeber of the IEICE (Japan), BCS (Bangladesh Computer Society, Bangladesh) and IEB (the Institution of Engineers, Bangladesh). His research interest includes image understanding and pattern recognition, Computer vision and Robotics etc.

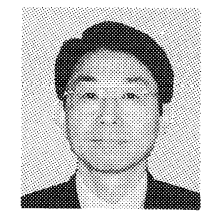

Noboru Ohnishi He received B.E., M.E., and Ph.D. degree in Electrical Engineering from Nagoya University at 1973, 1975, and 1984 respectively. From 1975-1986, he worked as a researcher in the Rehabilitation Engineering centre under the ministry of labour, Japan. In 1986, he joined as an Assistant Professor in the dept. of Electrical Engineering of Nagoay University. Currently, he is a professor of the dept. of Information Engineering at the same university. During his long journey in professional life, he was a visiting researcher(1992'93) in the laboratory of artificial intelligence at the Michigan University, team leader at the Biomimetic Control Research Center, RIKEN, Nagoya, Japan. He also hold many respectable positions at various professional bodies in Japan and he published many research papers(more than 90)in various international journals. For his technical creativity and ingenuity, he has been awarded by SICE society prizes in 1996 and 1999. His research interest includes brain analysis, modeling and brain support, computer vision and audition etc. 